\title{
Automatic Diagnosing of Suspicious Lesions in Digital Mammograms
}

\author{
Abdelali ELMOUFIDI ${ }^{1}$, K halid E1 Fahssi ${ }^{1}$, S aid J ai-andaloussi ${ }^{1}$, A bderrahim S ekkaki ${ }^{1}$, G wenole \\ Quellec $^{2}$ Mathieu Lamard ${ }^{2,3}$, Guy Cazuguel ${ }^{2,4}$ \\ ${ }^{1}$ Deprtment of Mathematics \& Computer Sciences, Faculty of sciences, Hassan II University of Casablanca, Casablanca, Morocco. \\ ${ }^{2}$ Inserm UMR 1101, Brest, F-29200 France. \\ ${ }^{3}$ Univ Bretagne Occidentale, Brest, F-29200 France. \\ ${ }^{4}$ Institut Mines-Telecom, Telecom Bretagne, UEB Dpt ITI, Brest, F-29200 France.
}

\begin{abstract}
Breast cancer is the most common cancer and the leading cause of morbidity and mortality among women's age between 50 and 74 years across the worldwide. In this paper we've proposed a method to detect the suspicious lesions in mammograms, extracting their features and classify them as Normal or Abnormal and Benign or Malignant for diagnosing of breast cancer. This method consists of two major parts: The first one is detection of regions of interest (ROIs). The second one is diagnosing of detected ROIs. This method was tested by Mini Mammography Image Analysis Society (Mini-MIAS) database. To check method's performance, we've used FROC (Free-Receiver Operating Characteristics) curve in the detection part and ROC (Receiver Operating Characteristics) curve in the diagnosis part. Obtained results show that the performance of detection part has sensitivity of $94.27 \%$ at 0.67 false positive per image. The performance of diagnosis part has $94.29 \%$ accuracy, with $94.11 \%$ sensitivity, $94.44 \%$ specificity in the classification as normal or abnormal mammogram, and has achieved $94.4 \%$ accuracy, with $96.15 \%$ sensitivity and $94.54 \%$ specificity in the classification as Benign or Malignant mammogram.

Index Terms-Breast cancer, Mammogram, Computer-aided diagnosis, Segmentation, Regions of interest, Support Vector Machine, FROC analysis, ROC analysis.
\end{abstract}

\section{INTRODUCTION}

Breast cancer is the most common cancer and the leading cause of morbidity and mortality among women's age between 50 and 74 years across the worldwide. Recent statistics have shown that one in 8 women in the United States and one in 10 women in Europe develop breast cancer during their lifetime $[1],[2]$. So, breast cancer is a major problem of public health, and the best strategy for the fight against breast cancer is early detection. For that reason, the mammography remains the best and most accurate tool for early detection of breast cancer [2],[3]. Reading and interpretation of mammogram is a crucial step. From where, Breast Imaging-Reporting and Data System (BI-RADS) of the American College of Radiology (ACR)[4], aims at providing a standardized classification system for reporting mammographic breast densities. Faced with the increase in the number of mammograms in recent decades, and the difficulty of reading and interpretation of mammograms, different research make the effort. Either, to automatically detect breast lesions through Computer Aided detection systems (commonly referred CADe). Either, To automatically interpret mammograms through Computer Aided Diagnostic Systems (commonly referred CADx). These systems are employed as a supplement to the radiologists' assessment.

Generally, the procedure to develop a Computer-AidedDiagnosis (CAD) system, for diagnosing of suspicious regions in mammograms takes place in four steps: 1) Preprocessing step: this step is to prepare the mammograms for the next steps of operations (segmentaton, classification); 2)Detection of regions of interest :This step is to analyze the mammogram and extract the necessary information, for example, segmentation which divides the mammogram into multiple segments, edge detection which finds the edges of objects and helps us to find regions of interest; 3) Features extraction and selection of ROIs detected: In this step, we can identify specific patterns, shapes, density and texture; 4) Classification of ROIs: The purpose of this step is to classify the mammograms as Normal or Abnormal and malignant or benign [5][6].

In this paper, we've proposed an automatic method to detect and diagnosing of suspicious lesions in mammogram. The proposed method is a very accurate technique for detecting and diagnosing breast cancer by using mammogram.

Obtained results show the efficiency of projected method and make sure chance of its use in rising breast cancer detection and the diagnosing.

Paper organization : The rest of paper organized as follows: Section I: An introduction ; Section II: Related work; Section III: Materials and method ; Section IV : Features generation and extraction; Section V : Our proposed research; Section VI : Results and performance of proposed method ; Section VII : Conclusion; and references are given at the end.

\section{RELATED WORK}

For detection and diagnosing of abnormalities in mammograms, A number of methods have been proposed, generally regrouped as: Statistical methods [7]; methods based wavelets [8][9]; Methods based Markov models [10]; Methods using machine learning[11], etc. Several researches have been published about computer breast cancer detection and diagnosis. For example, K. Ganesan et al. [12] presented an overview 
describe recent developments and advances in the field of computer-aided breast cancer diagnosis using mammograms. M. Veta et al.[13] presented an overview of methods that have proposed for the analysis of breast cancer histopathology images. Detection of ROIs is a capital step in development a computer-aided breast cancer diagnosis system. Many researchers have published on segmentation of breast tissue regions according to differences in density and texture, for detecting ROIs. For example, Adel et al. [14] used a method to segment mammograms into three distinct regions are : pectoral muscle, fatty regions, and fibroglandular regions using Bayesian techniques with Markov random field. Elmoufidi et al. [15][16] developed a method to Detect of ROIs in Mammograms using LBP algorithm, K-Means algorithm and GLCM algorithm. K. Hu et al. [2] published an approach to detect of suspicious lesions in mammograms by adaptive thresholding based on multiresolution. In other word, many methods have been used to feature extraction and classification. For example, Veena et al. [17] proposed a CAD System for Automatic Detection and Classification of Suspicious Lesions in Mammograms. Nasseer et al. [18] developed an algorithm for Classification of Breast Masses in Mammograms using SVM. L.Jelen et al. [19] developed a method for Classification of breast cancer malignancy using cytological images of fine needle aspiration biopsies. J. Malek et al. [20] proposed a system to Automatic Breast Cancer Diagnosis Based on GVFSnake Segmentation, Wavelet Features Extraction and Fuzzy Classification. Nra Szkely et al. [21] used A Hybrid System for Detecting Masses in Mammographic Images. [22] Used an approach for Mammogram Segmentation by Contour Searching and Massive Lesion Classification with Neural Network. S. Timp et al. [23] developed a Computer-aided diagnosis with temporal analysis to improve radiologists.

The CAD systems are powerful tools that could aid radiologists to lead better results in diagnosing a patient.

\section{Materials AND Method}

The proposed method checked by mini Mammography Image Analysis Society (mini-MIAS) database[24] and implemented using Seed Region Growing (SRG) algorithm, Local Binary Pattern (LBP) algorithm and support vector machine (SVM) classifier. The SRG to remove the pectoral muscle, the LBP to detect the regions of interest, and SVM to classify the mammograms as normal or abnormal and benign or malignant. SRG and LBP are two simples algorithms of segmentation and better choice for easy implementation. Using SVM as classifier because provide an effective and flexible framework from which to base CAD techniques for breast mammogram [25].

\section{A. Mammogram Database}

To checked the proposed method we've used the mini-Mammography Image Analysis Society (mini-MIAS) database[24]. The mammograms are in gray scale file format (Portable Grey Map - PGM), the size of every image is
$1024 \times 1024$ pixels, and resolution of 200 micron. This database composed of 322 mammograms of right and left breast, from 161 patients, where 207 mamograms diagnosed as normal and 115 mammograms as abnormal (22 images of CIRC - Well-defined/circumscribed masses, 19 images of SPIC - Spiculated masses, 19 images of ARCH - Architectural distortion, 15 images of ASYM - Asymmetry, 26 images of CALC - Calcification and 14 images of MISC - Other, illdefined masses) 52 mammograms malignant and 63 benign. Fig. 1 shows the different objects in the mammograms.

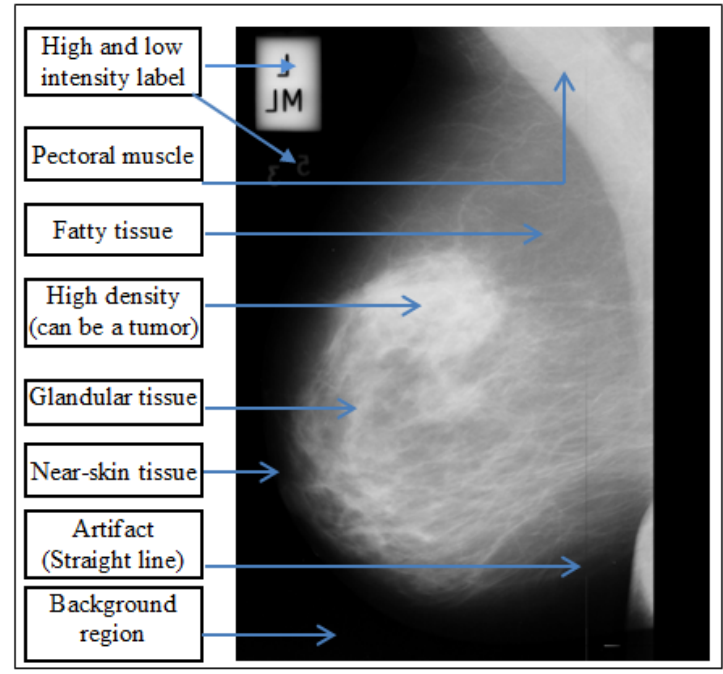

Fig. 1: The different elements in mammogram.

\section{B. Seed Region Growing (SRG)}

SRG algorithm for segmentation introduced by R. Adams et al. [25] is a simple method of segmentation which is free of tuning parameters and rapid. It's one of the better choice for easy implementation and applying it on a larger dataset. Seed region growing approach for image segmentation is to segment an image into regions with respect to a set of $\mathrm{N}$ seeds as presented in [12],[14] is discussed here.

\section{Local Binary Pattern (LBP)}

Local Binary Pattern (LBP) operator combines the characteristics of statistical and structural texture analysis. The LBP operator is used to perform gray scale invariant twodimensional texture analysis. The LPB operator labels the pixel of an image by Thresholding the neighborhood (i.e. $3 \times$ 3 ) of each pixel with the center value and considering the result of this Thresholding as a binary number [7],[26]. When all the pixels have been labeled with the corresponding LBP codes, histogram of the labels are computed and used as a texture descriptor. Formally, given a pixel at $\left(x_{c}, y_{c}\right)$, the resulting LBP can be expressed in decimal form as follows:

$$
L B P_{P, R}\left(x_{c}, y_{c}\right)=\sum_{P=0}^{P=1} S\left(i_{p}-i_{c}\right) 2^{P}
$$


where $: i_{c}$ and $i_{p}$ are, respectively, gray-level values of the central pixel and $\mathrm{P}$ surrounding pixels in the circle neighborhood with a radius $\mathrm{R}$, and function $\mathrm{s}(\mathrm{x})$ is defined as:

$$
S(x)=\left\{\begin{array}{l}
1, x \succeq 0 \\
0, x \prec 0
\end{array}\right.
$$

\section{Support Vector Machine (SVM)}

SVM classifier algorithm, developed from the machine learning community is a discriminative classifier formally defined by a separating hyperplane. The hyperplane is determined in such a way that the distance from this hyperplane to the nearest data points on each side, called support vectors, is maximal [27]. SVM classifiers can be extended to nonlinearly separable data with the help of kernel function application on the data to make them linearly separable [28]. An approach with wavelet SVM was discussed in [29]. Details about SVM, its application to diagnose of breast cancer was discussed in [26][30].

\section{Feature generation AND EXTRACTION}

Below a list of eighteen features selected to use as input parameters of SVM classifier for training and testing our proposed method.

a) Mean Value: $\mu$ represents the average of pixels in the segmented ROI.

$$
\mu=\frac{1}{M N} \sum_{i=1}^{M} \sum_{j=1}^{N} I(i, j)
$$
$\mathrm{MxN}$.

Where: $I(i, j)$ is the pixel value at point $(i, j)$ in ROI of size

b) Standard Deviation: $\sigma$ describes the dispersion within a local region.

$$
\sigma=\sqrt{\frac{1}{M N} \sum_{i=1}^{M} \sum_{j=1}^{N}(I(i, j)-\mu)^{2}}
$$

c) Entropy: H used to describe the distribution variation within ROI.

$$
H=-\sum_{k=1}^{L-1} P_{k} * \log _{2}\left(P_{k}\right)
$$

Where: $P_{k}$ is the probability of the $k^{t h}$ grey level, $\mathrm{L}$ is the total number of grey levels.

d) Skewness: $\mathrm{S}$ is a number characterizes the shape of the distribution.

$$
S=\frac{1}{M N} \sum_{i=1}^{M} \sum_{j=1}^{N}\left[\frac{I(i, j)-\mu}{\sigma}\right]^{3}
$$

Where: $I(i, j)$ the pixel value at point $(i, j), \mu$ the mean and $\sigma$ the standard deviation. e) Kurtosis: $\mathbf{K}$ measures the flatness of a distribution relative to a normal distribution.

$$
K=\left\{\frac{1}{M N} \sum_{i=1}^{M} \sum_{j=1}^{N}\left[\frac{I(i, j)-\mu}{\sigma}\right]^{4}\right\}-3
$$

f) Uniformity: $\mathbf{U}$ is a texture measure based on histogram :

$$
U=\sum_{k=0}^{L-1} P_{k}^{2}
$$

Where: $P_{k}$ the probability of the $k^{t h}$ grey level.

g) Sum Entropy: SE is a logarithmic function of the ROI in consideration.

$$
S E=-\sum_{i=2}^{2 N_{g}} p_{x+y}(i) \log \left\{p_{x+y}(i)\right\} .
$$

h) Sum Average: SA is found from the ROI in consideration and the size of the gray scale

$$
S A=\sum_{i=2}^{2 N_{g}} i p_{x+y}(i)
$$

i) Difference variance: $\mathrm{DV}$ is a variance measure between the ROI intensities calculated as a function of the SE calculated previously

$$
D V=\sum_{i=2}^{2 N_{g}}(i-S E)^{2} p_{x-y}(i)
$$

j) Difference entropy: DE is an entropy measure which provides a measure of no uniformity while taking into consideration a different measure obtained from the original image

$$
D E=-\sum_{i=2}^{2 N_{g}} p_{x-y}(i) \log \left\{p_{x-y}(i)\right\} .
$$

k) Inverse Difference Moments: IDM is a measure of the local homogeneity.

$$
I D M=\sum_{i} \sum_{j} \frac{1}{1+(i-j)^{2}} p(i, j) .
$$

l) Area: A is the sum of the number of all pixels (x) within segmented ROI.

$$
A=\sum_{x \in R O I} 1
$$


m) Perimeter: $\mathrm{P}$ is the length of a polygonal approximation of the boundary (B) of ROI:

$$
P=\sum_{x \in B} 1
$$

n) Convexity: $\mathbf{C}(\mathbf{S})$ is the ratio of the $\mathrm{ROI}$ area and its convex hull, the convex hull is the minimal area of the convex polygon that can contain the ROI:

$$
C(S)=\frac{A}{\operatorname{Area}(C H(S))} .
$$

Where: $\mathrm{S}$ is a $\mathrm{ROI}, \mathrm{CH}(\mathrm{S})$ is its convex hull and $\mathrm{A}$ is the ROI's area.

o) Compactness: C is a measure of ROI's shape, which indicates how much the ROI is compact :

$$
C=\frac{P^{2}}{4 \pi A} .
$$

Where : P the ROI's perimeter, A ROI's area.

p) Aspect Ratio: AR corresponds to the aspect ratio of the smallest window fully enclosing the ROI in both directions (see Fig.2):

$$
A R=\frac{D_{y}}{D_{x}}
$$

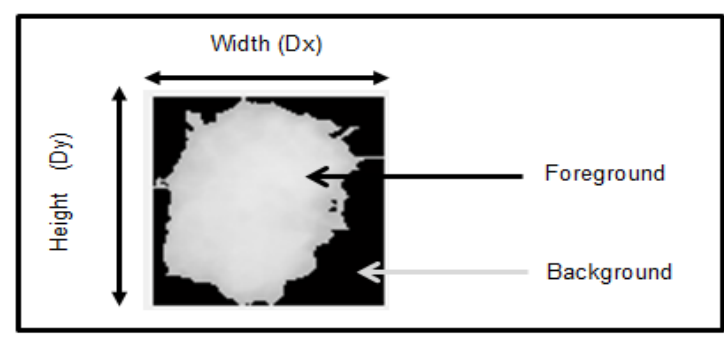

Fig. 2: Example of ROI window from which some features will be extracted.

Where: Dy the height, Dx the width of window in Fig.2

q) Area Ratio: The Area Ratio $\left(R_{-}\right.$Area $)$is defined by dividing the area of the segmented ROI in pixels by the area of the same window given in Fig.2 :

$$
R_{-} \text {Area }=\frac{\text { Area }_{-} R O I(\text { in pixels })}{\text { Area_window }(\text { in pixels })} .
$$

Where: Area_window $=\mathrm{Dx}^{*} \mathrm{Dy}, \mathrm{Dx}$ is the width's ROI and Dy is the height's ROI. The value of $R_{-}$Area will range from 0 to 1 . So, It takes small values for ROI with appendices and branches emitted from it, and larger values for more compacted and rounded objects. r) Perimeter Ratio: $R_{-}$Perim presents the ratio between the perimeter of the segmented ROI to the perimeter of the same rectangular window of fig. 2 , this can be written as:

$$
R_{-} \text {Perim }=\frac{\text { Perimeter } \_ \text {ROI }(\text { in pixels })}{\text { Perimeter_window }(\text { in pixels })} .
$$

\section{OUR PROPOSED RESEARCH}

In this paper, we've proposed a method for automatic detecting and diagnosis of suspicious lesions in mammograms. The proposed method consists four major blocks, namely: (1) Mammogram preprocessing performs three steps are: Remove the labels and additional objects in mammograms; Suppressed the background and the pectoral muscle; Eliminate the artifact, digital noise and contrast enhancement. (2) Segmentation and detection of ROIs, In this block, we've segmented and detected the ROIs are done using Local Binary Pattern . The details about LBP are discussed above. (3) Extraction and selection of features for each ROIs, in this case, we've combined the different types of features (size, density, shape, contrast and texture). (4) Classification of mammograms to normal or abnormal in the first time and benign or malignant in the second time using Support Vector Machine (SVM).

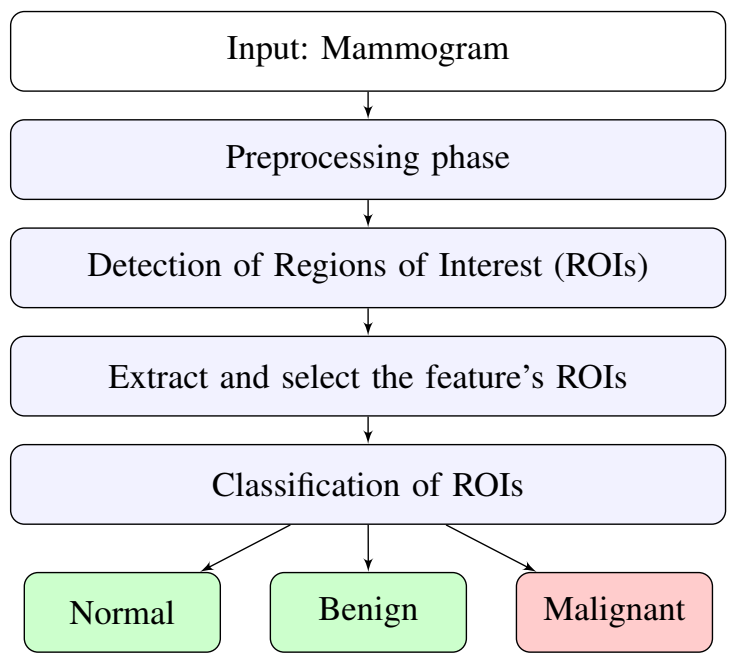

Fig. 3: Steps of the proposed method.

\section{A. Preprocessing}

The mammography can cause some additional objects at the resulting mammograms, like: artifact, noises, labels, etc. According to [31], the mammograms contain several sorts of noise and imaging artifacts. So, preprocessing step will be applied to get rid of the extra objects and enhance the standard of mammograms. Generally, the preprocessing step is to prepare the mammograms for the next steps, such as: segmentation of ROIs , selection and extraction of features of ROIs, and classification of ROIs. In this step, the aim is to extract only the breast profile region without additional 
objects, and without background. First, a threshold value is used to get rid of the labels and also the further objects within the mammograms. Second, we've used an automatic technique to take away further background, and detected mammogram orientation. From where, the pectoral muscle is within the top corner in right or left, the seed point of SRG is J[5,5] or J[5, $\mathrm{y}-5$ ], (were $\mathrm{J}$ : is the mammogram when the background has removed, $[\mathrm{x}, \mathrm{y}]=\operatorname{size}(\mathrm{J}))$ and we've used a minimal threshold value for giving a good result with all type of mammogram (Fatty , Fatty-glandular, Dense-glandular) . Third, we used 2D median filter in a 3-by-3 neighborhood connection to remove additional objects (artifact and noise). In addition, the mammogram is basically low contrast [1], so, we've applied a step of enhancement of contrast(see Fig.4).
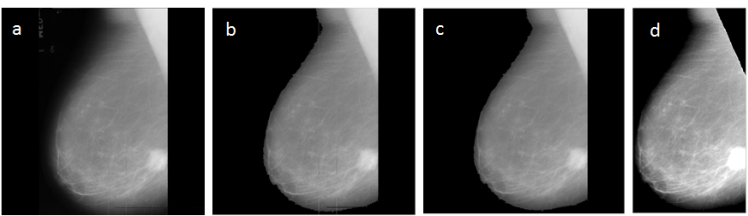

Fig. 4: Mammogram preprocessing: (a) Original mammogram, (b) Additional objects and Labels suppressed, (c) Noise and artifact removal, (d) Remove additional background and pectoral muscle. In addition, contrast enhancement.

\section{B. Detection of Regions of Interest (ROIs)}

Detection ROIs is a capital step in developing a CAD system, detecting several false positive result a weak system. To perform this task, we've implemented the Local Binary Pattern (LBP) algorithm for detecting the ROIs.

1) Experimental results of detection part:

a) Example 1: The first example deals with of normals mammograms. Fig.5.

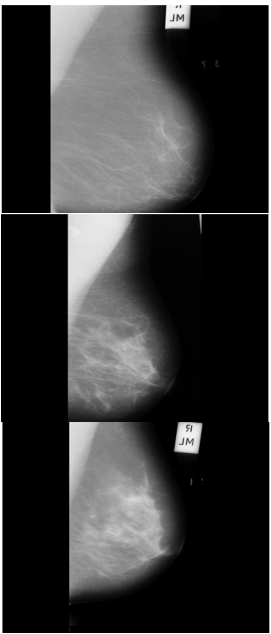

(a)

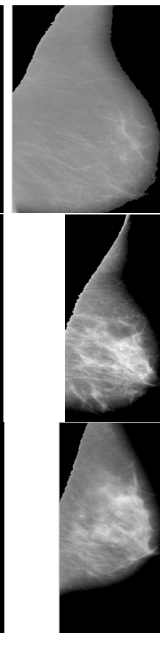

(b)

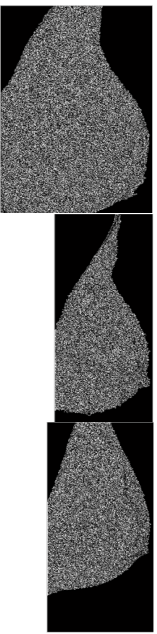

(c)

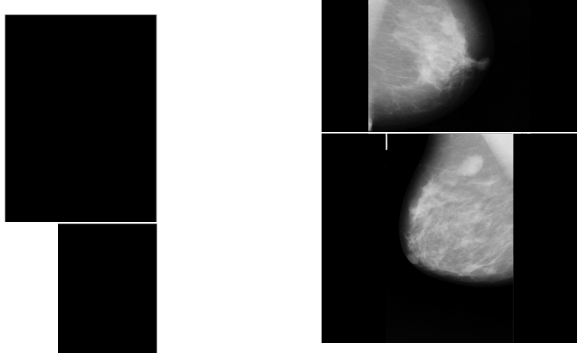

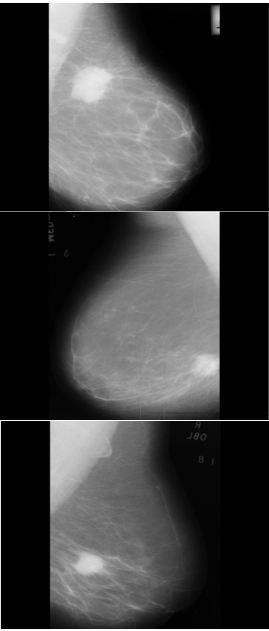

(a) (b)

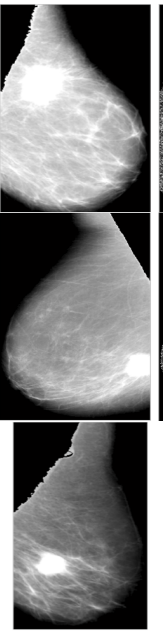

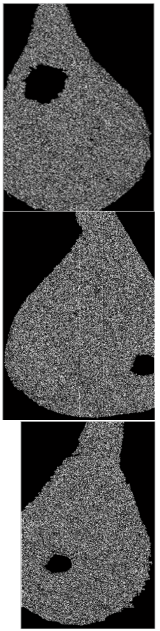

(c)

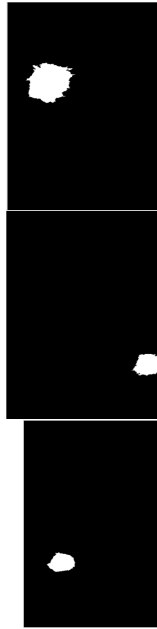

(d)
Fig. 6: ROIs detected contain the lesion without any false positive :(a) Original mammograms, (b) Mammograms after preprocessing, (c) LBP algorithm is applied,(d) regions of interest have detected .

c) Example 3: Mammograms which contains a single lesions has been correctly detected with other ROIs detected as false positive. Fig. 6 .

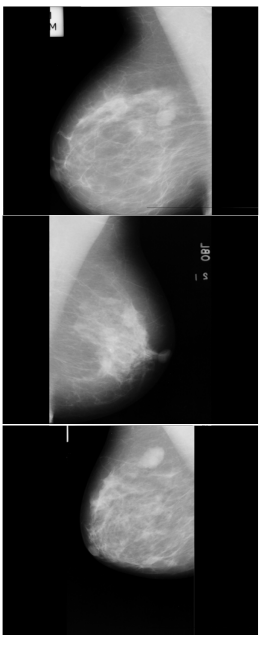

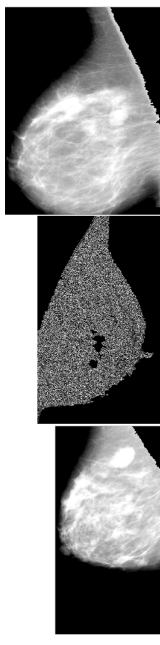

(b)

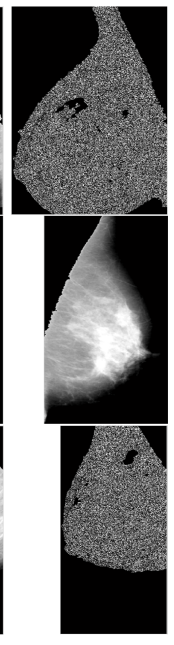

(c)

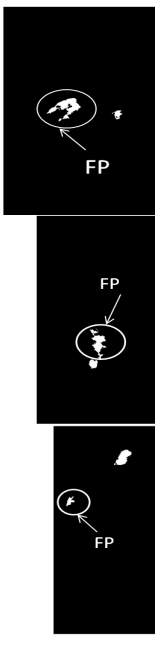

Fig. 7: ROIs detected contain the lesion and false positives :(a) Original mammograms, (b) Mammograms after preprocessing, (c) LBP algorithm is applied,(d)Regions of interst have detected.

2) Example of ROIs automatically detected: In the figure below, some ROIs automatically detected.

Fig. 5: Detection of ROIs :(a) Original mammograms, (b) Mammograms after preprocessing, (c) LBP algorithm is applied,(d) Any regions of interest have detected .

\section{Diagnosis of Regions of Interest (ROIs)}

After detection of regions of interest and extraction their features, the next step is to classify them as normal or 


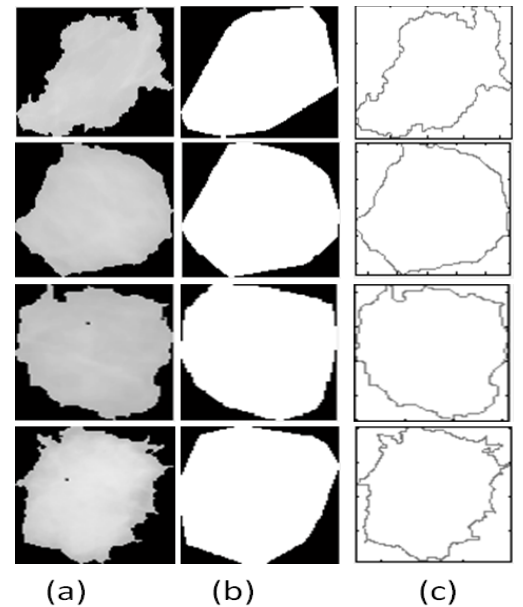

Fig. 8: Examples of ROIs detected: (a) ROIs detected, (b) convex hull of ROIs, (c) The contour of ROIs.

abnormal in the first time and as benign or malignant in the second time. One among the novelties of proposed method that a new technique to detect all suspected areas in mammogram ( not just the detection of lesions) and consider them as regions of interest (ROIs). If no regions of interest detected, the mammogram is normal. In the case of detection of multiple ROIs, we are going to separate them one by one and extracted their features separately (one by one), then diagnosing them. first, in the case all ROIs belong in the same mammogram are normal, then the mammogram is normal. Otherwise, the mammogram is abnormal. Second, in the case all ROIs belong in the same mammogram are benign, then the mammogram is benign. Otherwise, the mammogram is malignant. In addition, this algorithm is able to detect the masses and the calcification.

1) Experimental results of diagnosis part: Next three figures show details of the mentioned method. 1) Button "download" for downloading a new mammogram. 2) Button "Pre-processing" is to apply a preprocessing step on original mammogram (remove label, noise, pectoral muscle and additional background). 3) Button "Apply LBP" is to apply local binary pattern algorithm on the result mammogram after preprocessing step . 4) Button "Extract ROIs" is to extract all detected objects as ROIs. If we get just one ROI, only the button "ROI1" is going to enable. If we get two ROIs, the two buttons "ROI1" and "ROI2" are going to enable, and so on. 5) Button "ROI1" is to select the first ROI, the button "ROI2" is to select the second ROI, and so on. Button "Clac-features" is to extracte ROI's features selected in the previous step. 6) Button "add-feature" is to add the features in our database. 7) Button "Classify" is to classify the ROI selected to normal, benign or malignant. if the ROI selected is normal a white button appears on the screen containing the text normal, if the ROI selected is benign a green button appears on the screen contains the text benign, if the ROI selected is malignant a red button appears on the screen containing the text malignant. In addition, if we get many ROIs, we are going to classify them one by one, if all the ROIs are normals, the mammogram is normal. if there are at least one ROI benign and no ROI malignant, the mammogram is benign. If there are at least one ROI malignant, the mammogram is malignant.

a) Example 1: A normal mammogram correctly detection and diagnosis .

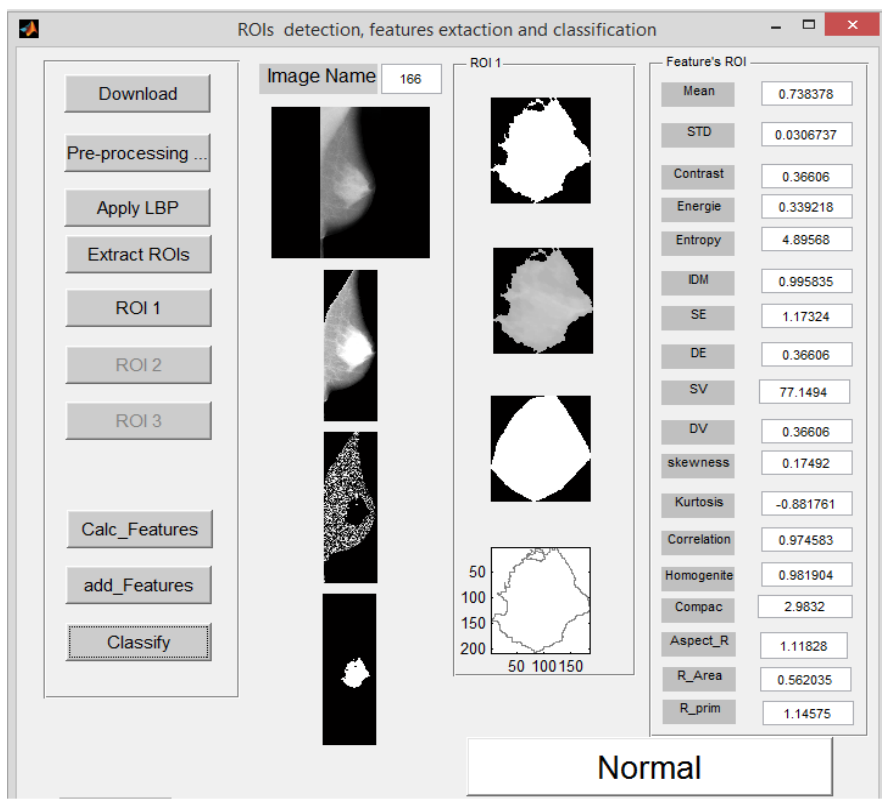

Fig. 9: Example 1: A normal mammogram Exactly diagnosis.

b) Example 2: A benign lesion correctly detection and diagnosis without false positive

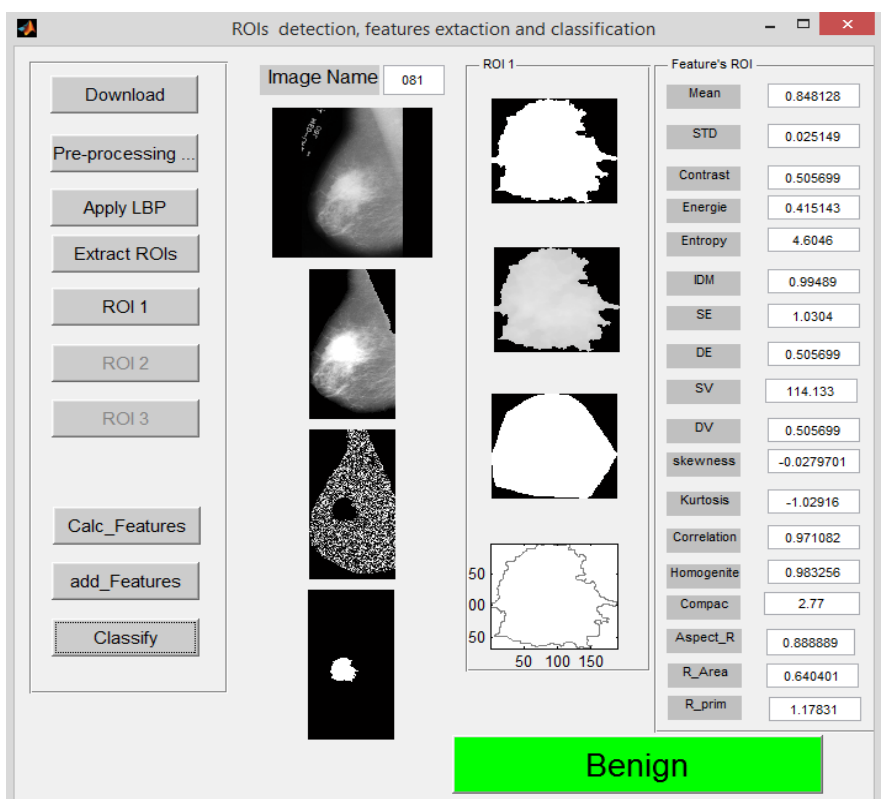

Fig. 10: Example 2: A Benign mammogram correctly diagnosing.

c) Example 3: A lesion malignant correctly detection/diagnosis without false positive 


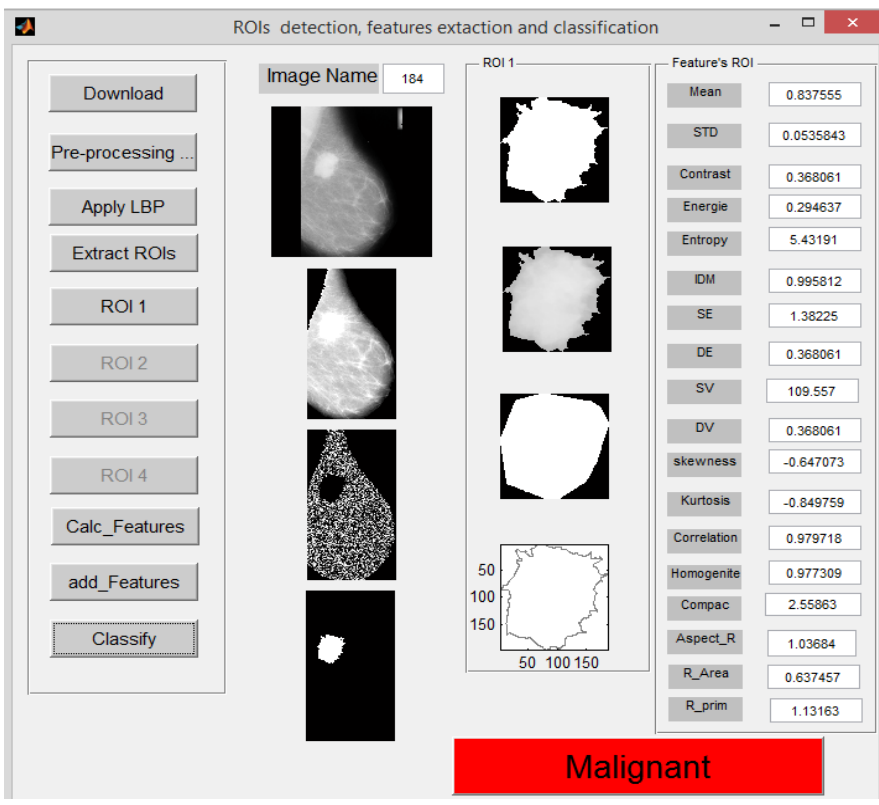

Fig. 11: Example 3: A Malignant mammogram correctly diagnosing..

\section{RESULTS AND PERFORMANCE}

Our global method were checked on 322 mammograms from mini-MIAS database. The detail about mini-MIAS database is given above.

\section{A. Performance of detection part}

Each segmentation and classification result needs evaluation of its performance. Generally, there are three types of performance evaluations of algorithms and approaches proposed for medical imaging processing (detection of regions of interest, segmentation and classification): The first type involves qualitative assessment, the second is quantitative assessment involving the ground truth evaluation and the third is a statistical evaluation [31].

Detected and selected the suspicious regions in mammogram is a crucial step in developing a CAD system, and detecting more regions d'interet as false positive, result a weak system. For that, we've considered a ROI correctly detected if its area is overlapped by at least of $75 \%$ from ground truth. We have obtained a good detection result, i.e., $100 \%$, for MISC and $95.45 \%$, for CIRC. The detection result of SPIC $(89.47 \%)$ is relatively reliable, because the overlapping of some SPIC is least of $75 \%$, hence, we considered as false negative. Generally, we've obtained a sensitivity of $94.27 \%$ at 0.67 False Positive per Image in the detection stage.

FROC curve, representing the True Positive Fraction (TPF) according False Positive per Image (FP/I) see the detail below:

$$
\text { False Positive per Image }=\frac{\text { Number of False Positive }}{\text { Number of image }}
$$

TABLE I: The obtained results grouped by anomaly classes .

\begin{tabular}{|c|c|c|}
\hline $\begin{array}{c}\text { Class of abnormality } \\
\text { present }\end{array}$ & $\begin{array}{c}\text { Number of } \\
\text { images }\end{array}$ & $\begin{array}{c}\text { Sensitivity } \\
(\%)\end{array}$ \\
\hline Normal & 207 & $94.21 \%$ \\
\hline CIRC & 22 & $95.45 \%$ \\
\hline SPIC & 19 & $89.47 \%$ \\
\hline ARCH & 19 & $94.73 \%$ \\
\hline ASYM & 15 & $93.3 \%$ \\
\hline CALC & 26 & $92.31 \%$ \\
\hline MISC & 14 & $100 \%$ \\
\hline Total & 322 & $94.21 \%$ \\
\hline
\end{tabular}

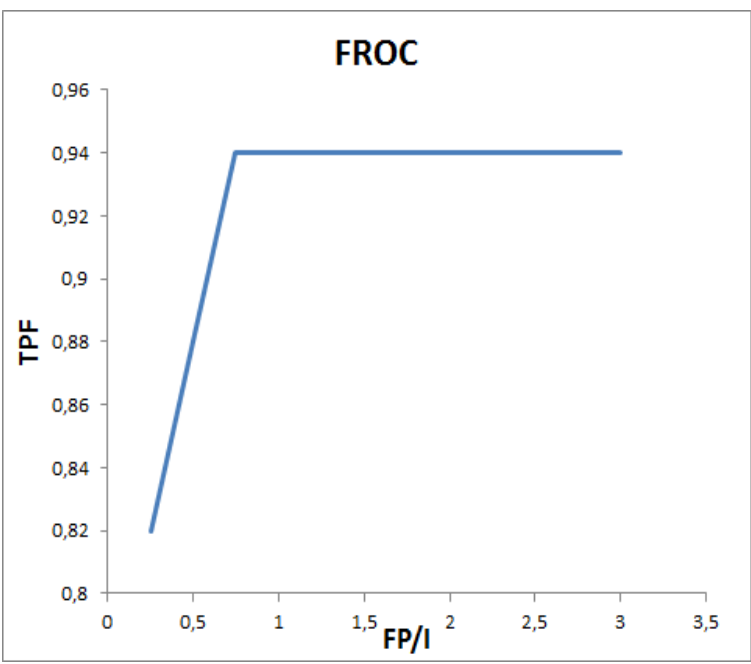

Fig. 12: FROC curve.

The evaluation procedure is as following: the database is divided into two parts: the first one for training contains the half of database (161 mammograms from 322 mammograms) selected aleatory, the second one for testing contains the rest of database ( 161 mammograms) the detail of the database distribution between training and testinig is given below:

TABLE II: Images's number used to train SVM Classifier.

\begin{tabular}{|c|c|c|c|c|}
\hline Image & Normal & Abnormal & Benign & Malignant \\
\hline Training & 104 & 57 & 31 & 26 \\
\hline Testing & 103 & 58 & 32 & 26 \\
\hline Total & 207 & 115 & 63 & 52 \\
\hline
\end{tabular}

\section{B. Performance of diagnosing part}

We have evaluated the performance of proposed method by calculating of accuracy, sensitivity and specificity for normal or abnormal case and benign or malignant case.

TABLE III: Diagnosing accuracy of normal or abnormal cases

\begin{tabular}{|c|c|c|c|c|}
\hline \multirow{2}{*}{} & \multicolumn{2}{|c|}{ Training } & \multicolumn{2}{c|}{ Testinig } \\
\cline { 2 - 5 } & Normal & Abnormal & Normal & Abnormal \\
\hline Normal & $(102) \mathrm{TP}$ & $(2) \mathrm{FP}$ & $(100) \mathrm{TP}$ & $(4) \mathrm{FP}$ \\
\hline Abnormal & $(2) \mathrm{FN}$ & $(53) \mathrm{TN}$ & $(3) \mathrm{FN}$ & $(52) \mathrm{TN}$ \\
\hline
\end{tabular}

Diagnosing part of our method has achieved $94.29 \%$ accuracy, with $94.11 \%$ sensitivity and $94.44 \%$ specificity. Fig. 12 shows the ROC curve of the proposed method. 
TABLE IV: Diagnosing accuracy of benign or malignant cases

\begin{tabular}{|c|c|c|c|c|}
\hline \multirow{2}{*}{} & \multicolumn{2}{|c|}{ Training } & \multicolumn{2}{c|}{ Testing } \\
\cline { 2 - 5 } & Benign & Malignant & Benign & Malignant \\
\hline Benign & $(30) \mathrm{TP}$ & $(1) \mathrm{FP}$ & $(29) \mathrm{TP}$ & $(2) \mathrm{FP}$ \\
\hline Malignant & $(2) \mathrm{FN}$ & $(24) \mathrm{TN}$ & $(3) \mathrm{FN}$ & $(23) \mathrm{TN}$ \\
\hline
\end{tabular}

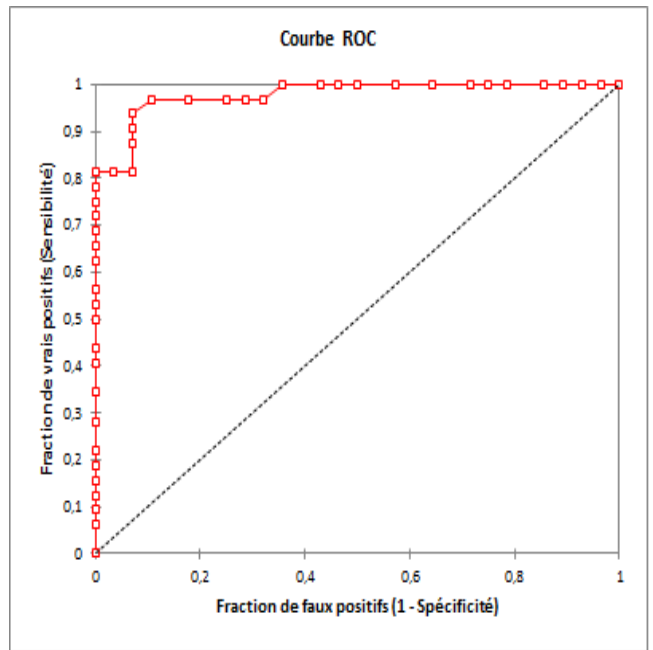

Fig. 13: ROC curve.

\section{Comparison our method with existing papers.}

TABLE V: Comparison our method's Performance with articles recently published.

\begin{tabular}{|c|c|c|}
\hline Authors & Method proposed & Accuracy \\
\hline $\begin{array}{c}\text { K. Hu } \\
\text { et al.[2] }\end{array}$ & $\begin{array}{c}\text { Detection of suspicious lesions } \\
\text { in mammograms }\end{array}$ & $91.3 \%$ \\
\hline $\begin{array}{c}\text { Veena } \\
\text { et al.[17] }\end{array}$ & $\begin{array}{c}\text { Detection \& Classification of } \\
\text { Suspicious Lesions in Mammograms }\end{array}$ & $92.13 \%$ \\
\hline $\begin{array}{c}\text { Nasseer } \\
\text { et al.[18] }\end{array}$ & $\begin{array}{c}\text { Classification of Breast Masses } \\
\text { in Digital Mammograms }\end{array}$ & $93.069 \%$ \\
\hline $\begin{array}{c}\text { K. Ganesan } \\
\text { et al.[27] }\end{array}$ & $\begin{array}{c}\text { Classification of Mammograms } \\
\text { Using Trace Transform Functionals }\end{array}$ & $92.48 \%$ \\
\hline $\begin{array}{c}\text { Our } \\
\text { method }\end{array}$ & $\begin{array}{c}\text { Automatic Diagnosing of Suspicious } \\
\text { Lesions in Digital Mammograms }\end{array}$ & $94.29 \%$ \\
\hline
\end{tabular}

\section{Conclusion}

In this paper, an automatic algorithm to breast cancer detection and diagnosing is implemented using the MATLAB environment. our algorithm's performance has evaluated using FROC curve in detection part and ROC curve in diagnosis part. Obtained results show the efficiency of this method and comparable to different solutions. The proposed algorithm will contribute to determination of main drawback in diagnostic procedure mammogram, such as: detection and diagnosing of the masses and also the calcification. The efficiency of the planned method confirms possibility of its use in up the Computer-Aided Diagnosis system.

\section{REFERENCES}

[1] A. ELMOUFIDI Member IEEE et al., "Automatically Density Based Breast Segmentation for Mammograms by using Dynamic K-means
Algorithm and Seed Based Region Growing,” I2MTC 2015 - International Instrumentation and Measurement Technology Conference , PISA, ITALY, MAY 11-14, 2015.

[2] K. Hu et al., "Detection of suspicious lesions by adaptive thresholding based on multiresolution analysis in mammograms," IEEE Trans on Instrumentation and Measurement, vol. 60, no. 2, pp. 462-472, 2010.

[3] A. Ferrero Fellow IEEE et al., "Uncertainty evaluation in a fuzzy classifier for microcalcifications in digital mammography," I2MTC 2010 International Instrumentation and Measurement Technology Conference Austin, TX, 3-6 May 2010.

[4] American College of Radiology. American College of Radiology Breast Imaging Reporting and Data System (BIRADS). $4^{\text {th }}$ ed., American College of Radiology, Reston, VA 2003.

[5] A.Jalalian et al., "Computer-aided detection/diagnosis of breast cancer in mammography and ultrasound," Clinical Imaging, 37 ,2013 420426.

[6] S. Shirmohammadi and A. Ferrero, "Camera as the Instrument: The Rising Trend of Vision Based Measurement," IEEE Instrumentation and Measurement Magazine, Vol. 17, No. 3, June 2014, pp. 41-47. DOI: 10.1109/MIM.2014.6825388

[7] H. Chan et al., "Computerized analysis of mammographic micro calcifications in morphological and feature spaces," Medical Physics, vol.25, no.10, pp.2007-2019, 1998

[8] A. Mencattini et al., "Mammographies images enhancement and denoising for breast cancer detection using dyadic wavelet processing," IEEE Trans. Instrumentation Measure., 57: 1422-1430. DOI: 10.1109/TIM. 2007. 915470.

[9] T. Wang and N. Karayiannis, "Detection of microcalcification in digital mammograms using wavelets," IEEE Trans. Medical Imaging, vol.17, no.4, pp.498-509, 1998.

[10] H. Li et al., "Marcov random field for tumor detection in digital mammography," IEEE Trans. Medical Imaging, vol.14, no.3, pp.565576, 1995.

[11] Drew P.J.Monson (J.R.T), "Artificial neural networks", Surgery volume 127, 2000, pp. 3-11.

[12] Karthikeyan Ganesan et al., "Computer-Aided Breast Cancer Detection Using Mammograms," IEEE Reviews in biomedical engineering, vol. 6, 2013.

[13] M. Veta, et al. "Breast cancer histopathology image analysis: a review.", IEEE transactions on bio-medical engineering, vol. 61, no. 5, pp. 140011, May 2014.

[14] Adel et al. , "Statistical Segmentation of Regions of Interest on a Mammographic Image," EURASIP Journal on Advances in Signal Processing 2007, Article ID 49482, 1-8 2007.

[15] Abdelali Elmoufidi et al.,"Detection of Regions of Interest in Mammograms by Using Local Binary Pattern, Dynamic K-Means Algorithm and Gray Level Co-occurrence Matrix," 2014 Fifth International Conference on Next Generation Networks and Services (NGNS'14) 28-30 May 2014, Casablanca, Morocco.

[16] A. Elmoufidi et al, "Detection of Regions of Interest in Mammograms by Using Local Binary Pattern and Dynamic K-Means Algorithm," International Journal of Image and Video Processing: Theory and Application Vol. 1, No. 1, 30 April 2014. ISSN: 2336-0992.

[17] Veena, et al., "CAD Based System for Automatic Detection et Classification of Suspicious Lesions in Mammograms," International Journal of Emerging Trends et Technology in Computer Science (IJETTCS) ISSN 2278-6856, Volume 3, Issue 4 July-August 2014.

[18] Nasseer M. Basheer et al., "Classification of Breast Masses in Digital Mammograms Using Support Vector Machines," International Journal of Advanced Research in Computer Science and Software Engineering ISSN: 2277 128X, Volume 3, Issue 10, October 2013.

[19] L. Jelen et al., "Classification of breast cancer malignancy using cytological images of fine needle aspiration biopsies," int. j. appl. math. comput. sci., 2008, vol. 18, no. 1, 7583 doi: 10.2478/v10006-008-0007-x.

[20] Jihene Malek et al., "Automated Breast Cancer Diagnosis Based on GVF-Snake Segmentation, Wavelet Features Extraction and Fuzzy Classification," J Sign Process Syst. DOI: 10.1007/s11265-008-0198-2

[21] N.Szkely et al., "A Hybrid System for Detecting Masses in Mammographic Images," IEEE Trans. Instrum. Meas., vol. 55, no. 3 June 2006.

[22] Cascio D. et al., "Mammogram Segmentation by Contour Searching and Massive Lesion Classification with Neural Network," Institute of Electrical and Electronic Engineering (IEEE), 2006.

[23] S. Timp et al., "Computer-aided diagnosis with temporal analysis to improve radiologists' interpretation of mammographicmass lesions," 
IEEE Trans. Inform. Technol. Biomedicine, vol. 14, no. 3, pp. 803808, May 2010.

[24] J. Suckling et al., "The Mammographic Image Analysis Society digital mammogram database," Exerpta Medica, International Congress Series 1069 pp. 375-378., 1994.

[25] Jacob Levman, "Classification of Dynamic Contrast-Enhanced Magnetic Resonance Breast Lesions by Support Vector Machines", IEEE Transactions On Medical Imaging, Vol. 27, No. 5, May 2008.

[26] H. X. Liu, et al., "Diagnosing Breast Cancer Based on Support Vector Machines", J. Chem. Inf. Comput. Sci. 2003, 43, 900-907.

[27] K. Ganesan et al., "One-Class Classification of Mammograms Using Trace Transform Functionals", IEEE Transactions on Instrumentation and Measurement, Vol. 63, No. 2, February 2014.

[28] K. R. Muller, et al. "An introduction to kernel based learning algorithms", IEEE Trans.Neural Netw., vol. 12, no. 2, pp. 181201, Mar. 2001.

[29] M. Shen et al., "A prediction approach for multichannel EEG signals modeling using local wavelet SVM", IEEE Trans. Instrum. Meas., vol. 59, no. 5, pp. 14851492, May 2010.

[30] L. Wei, et al., "A study on several machine-learning methods for classification of malignant and benign clustered microcalcifications," IEEE Trans. Med. Imag., vol. 24, no. 3, pp. 371380, Mar. 2005.

[31] Stylianos.D et al., "A fully automated scheme for mammographic segmentation and classification based on breast density and asymmetry," computer methods and programs in biomedicine 2011, 47-63. 